\title{
Planar pn heterojunction organic light-emitting diodes
}

\author{
Dongge $\mathrm{Ma}^{*}$ \\ State Key Laboratory of Polymer Physics and Chemistry; Changchun Institute of Applied Chemistry, Chinese Academy of Sciences, \\ Changchun 130022, China
}

Received November 26, 2015; accepted December 22, 2015; published online January 8, 2016

Citation: Ma DG. Planar pn heterojunction organic light-emitting diodes. Sci China Chem, 2016, 59: 205, doi: 10.1007/s11426-016-5555-6

Organic light-emitting diodes (OLEDs) are being increasingly applied in flat-panel displays, such as television and mobile phones, and have great potential to become next generation solid-state lighting sources. Along with the commercialization of OLED products, scientists are devoting continuous efforts to investigate novel physical mechanisms and device configurations of OLEDs, targeting the simultaneous development of high performance (e.g. high electric-to-light conversion efficiency, good chromatic parameters to meet various requirements, and sufficiently long lifetime) and low-cost organic electroluminescent (EL) devices. One of the most attractive strategies is to construct OLEDs with low cost all-fluorescent materials, so that high cost phosphorescent materials containing rare or noble metals are not involved in device fabrication.

Prof. Shi-Jian Su's group at South China University of Technology in Guangzhou, China, has now proposed a simple strategy towards developing high-performance fluorescent OLEDs [1]. The designed devices are merely composed of a stacked p-type hole-transport layer and an n-type electron- transport layer (Figure 1(a)), resulting in a planar pn heterojunction configuration similar to their inorganic counterparts, namely light emitting diodes (LEDs). In this novel device, the excitons are generated and decay at the pn junction interface due to the synergetic interaction of p-type and n-type materials, resulting in light emission.

The most distinguishing feature of pn-OLEDs over conventional OLEDs is the absence of an independent light emission layer (EML) in the device. Due to the absence of an EML, the device structure of pn-OLEDs is greatly sim-

*Corresponding author (email: mdg1014@ciac.ac.cn) plified, which is significantly beneficial to reducing manufacturing processes and fabricating cost. In addition, the emission color of these EML-free planar pn heterojunction OLEDs could be tuned by solely changing the p-type material. Currently, the maximum obtained external quantum efficiencies (EQEs) for yellow and green OLEDs are as high as $12.0 \%$ and $10.1 \%$, respectively; this is due to simultaneous harvesting of singlet and triplet excitons for radiative transition. Besides, an extremely low driving voltage, where the threshold voltage for electroluminescence is even comparable with the theoretical minimum value of the photon energy $(h v)$ divided by electron charge (e), could also be realized for the resulting devices. Therefore, it is predicted that pn heterojunction OLEDs will show great potential in practical applications of display and lighting due to the advantages of a simplified structure, an extremely low driving voltage, and high efficiency. (a)

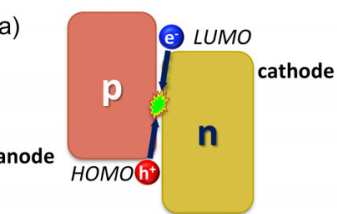

(b)

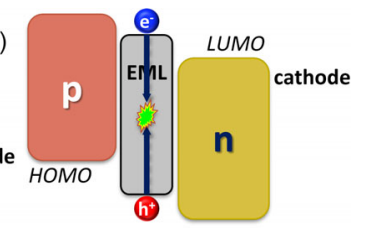

Figure 1 Schematic diagrams of (a) planar pn heterojunction OLED (pn-OLED) without a light emission layer (EML) and (b) conventional OLED with an EML.

Conflict of interest The authors declare that they have no conflict of interest.

1 Chen DC, Xie GZ, Cai XY, Liu M, Cao Y, Su SJ. Adv Mater, 2015, DOI: $10.1002 /$ adma.201504290 